Témoigner Témoigner. Entre histoire et mémoire

Getuigen Revue pluridisciplinaire de la Fondation Auschwitz

$122 \mid 2016$

Révisionisme et négationisme

\title{
Quand le corps est oublié. Les séquelles physiques du génocide pour les rescapés
}

Rwanda, épisode 6

When the body is forgotten: the physical consequences of genocide for

the survivors. Rwanda, episode 6

\section{Rémi Korman}

\section{(2) OpenEdition}

\section{Journals}

Édition électronique

URL : https://journals.openedition.org/temoigner/4673

DOI : $10.4000 /$ temoigner.4673

ISSN : 2506-6390

Éditeur :

Éditions du Centre d'études et de documentation Mémoire d'Auschwitz, Éditions Kimé

Édition imprimée

Date de publication : 2 mai 2016

Pagination : 162-165

ISSN : 2031-4183

Référence électronique

Rémi Korman, «Quand le corps est oublié. Les séquelles physiques du génocide pour les rescapés », Témoigner. Entre histoire et mémoire [En ligne], 122 | 2016, mis en ligne le 30 septembre 2021, consulté le 05 janvier 2022. URL : http://journals.openedition.org/temoigner/4673 ; DOI : https://doi.org/ $10.4000 /$ temoigner.4673 


\section{q mémoriel

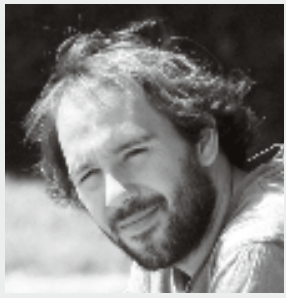 \\ $\rightarrow$ Par Rémi Korman, EHESS (École des Hautes Etudes en \\ QUAND LE CORPS EST OUBLIÉ LES SÉQUELLES PHYSIQUES DU GENOCIDE POUR LES RESCAPÉS}

Sciences Sociales). Il effectue des recherches universitaires sur la mémoire du génocide des Tutsi au Rwanda où il se rend régulièrement. Il développe en parallèle des projets de transmission publique de cette mémoire avec l'association lbuka à Lyon.

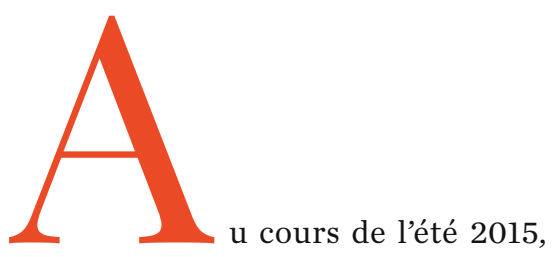

la mort est venue frapper deux jeunes rescapés du génocide contre les Tutsi vivant en diaspora, Berthe Kayitesi et Léandre Rugali Murara. Décédée d'une rupture d'anévrisme le 23 juin 2015, Berthe Kayitesi avait 36 ans. Elle vivait au Canada, y avait effectué ses études universitaires et récemment soutenu une thèse de doctorat (Kayitesi, 2014). Elle était particulièrement connue pour son engagement associatif et avait publié un ouvrage portant sur le rôle des enfants chefs de ménage au Rwanda après le génocide (Kayitesi, 2009). Léandre Rugali Murara vivait lui en Suisse depuis le début des années 2000 et travaillait pour la Fondation FoyerHandicap. Âgé de 35 ans, il est mort le 7 juillet 2015 par noyade, à la suite d'une crise d'épilepsie.

Si la mort de Berthe Kayitesi apparaît comme naturelle, tout aussi déchi- rant que puisse apparaître ici ce mot pour une jeune femme de 36 ans, il n'en est pas de même pour Léandre Rugali Murara. Pour de nombreux rescapés, sa noyade est une des conséquences du génocide, celui-ci étant devenu épileptique après 1994, en raison de nombreux coups qui lui avaient été alors infligés.

Dans cet article, je souhaite simplement proposer une très courte réflexion sur un thème largement négligé, celui des conséquences physiques du génocide et de la probable surmortalité des rescapés après 1994.

\section{"L'EMPIRE DU TRAUMATISME "}

Dans un ouvrage, depuis devenu référence, publié en 2007, Didier Fassin et Richard Rechtman interrogent la place accordée à la question du traumatisme et l'essor de ce concept depuis plusieurs dizaines d'années (Fassin et Rechtman, 2007). Le titre de l'ouvrage, 


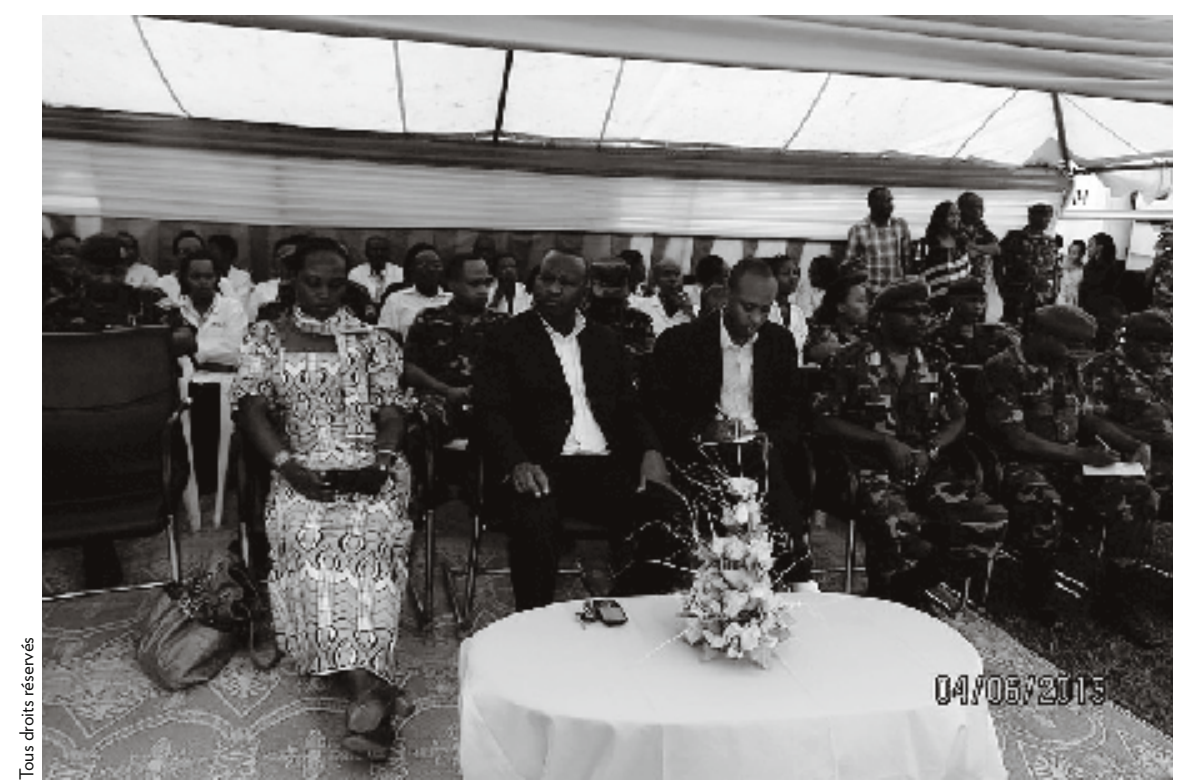

L'empire du traumatisme, vise à interroger la place, parfois démesurée, accordée maintenant à cette question après tout événement ou toute crise. Au Rwanda, le génocide a évidemment entraîné de nombreuses conséquences psychologiques, en particulier pour les rescapés, et il existe des formes particulières de traumatisme du génocide (Gishoma, 2014). Mais on remarque que la littérature scientifique sur ces enjeux est extrêmement abondante : des dizaines de thèses et d'ouvrages, des centaines de mémoires étudiants, ainsi que des rapports d'ONG.

À cette abondance de travaux s'oppose, en comparaison, un désintérêt de fait sur les séquelles physiques du génocide. Cette situation est d'autant plus étonnante que la recherche historique actuelle s'intéresse largement aux types de violences faites aux corps des victimes au cours du génocide (Audoin-
Rouzeau et Dumas, 2014). En l'absence de véritable recherche, plusieurs questions préparatoires peuvent être posées : existent-ils des «pathologies du génocide» ? Comment les séquelles physiques du génocide ont été traitées au Rwanda depuis 1994? Quels sont les enjeux actuels?

De façon générale, les rapports sur les rescapés du génocide mentionnent depuis 1994 quatre grands groupes de victimes. Tout d'abord les orphelins (imfubyi), puis les veuves (abapfakazi), ensuite les traumatisés (abahungabanye) et enfin les handicapés (abamugaye). Si les trois premiers groupes ont été largement mentionnés par les chercheurs, ce n'est pas le cas du dernier. Il existe peu de statistiques précises sur ce point. Depuis 1994, plusieurs recensements de rescapés du génocide ont été réalisés. Le premier fut mené en 1998 par le MIJESCAFOP (ministère de la •••
_ Medical Army week for Genocide survivors, 2013 
Jeunesse, des Sports, de la Culture et de la Formation professionnelle), le second en 2008 par le MINALOC (ministère de l'Administration locale, de la Bonne Gouvernance et des Affaires sociales) et l'Institut National des Statistiques du Rwanda. Ces recensements visaient à comptabiliser le nombre de rescapés vivant au Rwanda, mais aussià connaître leurs conditions de vie matérielles et médicales. Les problèmes méthodologiques entourant ces rapports ne permettent pas d'avoir des données fiables. Il est difficile alors de savoir s'il existe ou non une surmortalité chez les rescapés, en comparaison du reste de la population, mais aussi de connaître les besoins spécifiques concrets.

À cette invisibilisation statistique s'ajoute une invisibilisation dans la sphère publique des personnes rendues infirmes par le génocide, ou encore de ce que l'on peut nommer en reprenant le vocabulaire relatif à la Première Guerre mondiale, des «gueules cassées ». Au sein de la littérature de témoignage, l'ouvrage de Révérien Rurangwa apparaît alors comme une exception, en donnant une place importante à l'importance des conséquences physiques du génocide (Rurangwa, 2006).

\section{LA LENTE PRISE EN CHARGE MÉDICALE DES RESCAPÉS}

Si nous savons comment depuis 1994 a été prise en charge la question du traumatisme des rescapés du génocide, avec par exemple la création dès 1995 du CNT, le Centre National du Traumatisme basé à Kigali, comment ont été prises en charges les séquelles physiques du génocide?

Rappelons tout d'abord que la disparition d'une partie importante du personnel médical a entraîné un dysfonctionnement du système de santé postgénocide. Nous savons qu'un nombre important d'ONG ont participé aux soins de rescapés au cours des années 1990, ce fut le cas pour Révérien Rurangwa. L'association de rescapés du génocide Ibuka a aussi participé de son côté au recensement de rescapés nécessitant des soins et s'est occupé de transmettre les informations au FARG, le Fonds d'Aide aux Rescapés du Génocide, lors de sa création en 1998. Cette dernière institution, dont le budget représente $5 \%$ du budget annuel de l'État rwandais, a connu malheureusement de nombreux dysfonctionnements, qui ont entravé de façon régulière le bon accès aux soins des victimes.

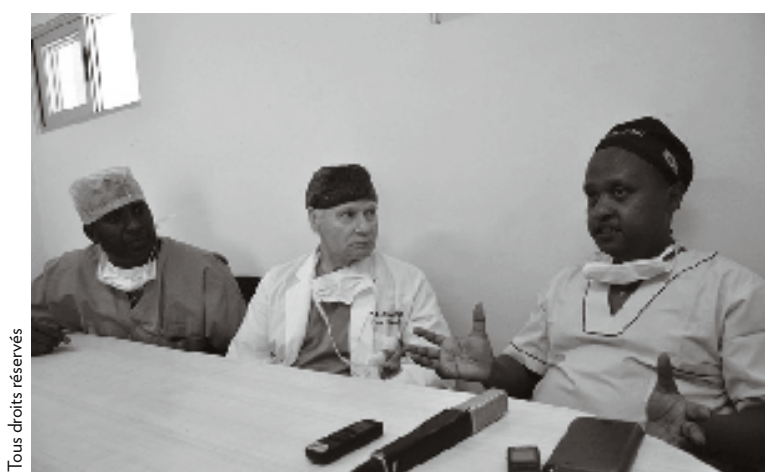

_ Lieutenant Colonel Dr. Furaha, Dr. Peter Adamson et le Dr. Ifepo Sofola parlant aux médias à l'hôpital militaire rwandais, 2014 
En raison de la mauvaise gestion du fonds, et des dettes accumulées, la question de la santé fut déconsidérée pendant plusieurs années, en comparaison des enjeux de logement ou encore des frais de scolarité. Aggée Shyaka Mugabe note d'ailleurs que de 1998 à 2005, seulement 107 transferts de rescapés à l'étranger pour raisons médicales furent autorisés (Shyaka, 2009, 153). De fait, au cours des quinze années qui suivirent le génocide, de nombreux rescapés ne furent pas soignés, malgré d’importantes séquelles. Le cas le plus dramatique est probablement celui des femmes violées et contaminées par le SIDA. Beaucoup sont ainsi mortes du génocide à rebours et d'un second abandon de la communauté internationale.

Cependant, depuis le début de la décennie actuelle, ont été mis en place d'importants projets de traitement médical, mais aussi de chirurgie reconstructrice en faveur des rescapés. En 2012, a ainsi été instituée par l'armée rwandaise, dite RDF pour Rwandan Defence Forces, la Medical Army week for Genocide survivors. Cette initiative a été lancée en partenariat avec le FARG et vise à aller soigner les rescapés dans chaque district. En parallèle a été ouvert, en 2012, au sein de l'hôpital militaire de Kanombe, à Kigali, un service spécialisé en chirurgie plastique et réparatrice. Il existe actuellement au Rwanda un unique spécialiste en chirurgie plastique, le lieutenant Charles Furaha, mais la discipline tend à se développer (Deutsche Welle, 2013).

L'évolution actuelle très positive du système de santé rwandais, avec par exemple la création d'une mutuelle de santé généralisée, laisse espérer une meilleure prise en compte des enjeux de santé pour les rescapés du génocide.
Cependant, il apparaît nécessaire que les séquelles physiques du génocide soient mieux considérées d'un point de vue intellectuel et évidemment médical. Si le Rwanda en tant que pays s'est reconstruit, il est important de rappeler que le génocide a entraîné des conséquences qui ne sont pas que mentales, mais aussi physiques.

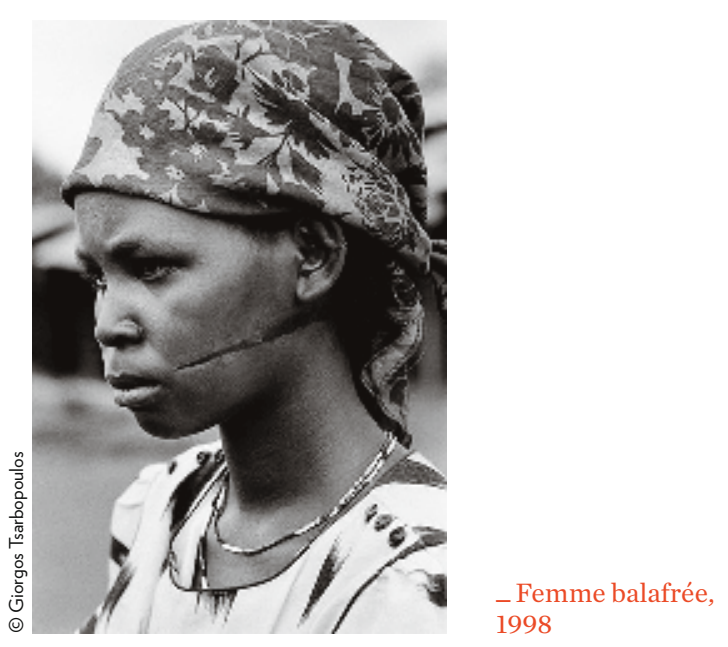

\section{BIBLIOGRAPHIE}

- Audoin-Rouzeau, Stéphane et Dumas, Hélène (avril 2014/2) : «Le génocide des Tutsi rwandais vingt ans après », Vingtième Siècle. Revue d'histoire, n ${ }^{\circ} 122$, p. 3-16.

- Deutsche, Welle, Émission santé, "Chirurgie réparatrice pour les séquelles du génocide », 9 janvier 2013.

- Fassin, Didier et Rechman, Richard (2007) : L'empire du traumatisme. Enquête sur la condition de victime, Paris, Flammarion.

- Gishoma, Darius (2014) : Crises traumatiques collectives d'ihahamuka lors des commémorations du génocide des Tutsi : aspects cliniques et perspectives thérapeutiques, Louvain-la-Neuve.

- Kayitesi, Berthe (2009) : Demain ma vie. Enfants chefs de famille dans le Rwanda d'après, Paris, Laurence Teper.

- Kayitesi, Berthe (2014) : Conséquences et résilience liées à l'apprentissage et à la recherche sur le génocide des Tutsis : Cas des étudiants et des professeurs, Université d'Ottawa.

- Rurangwa, Révérien (2006) : Génocidé, Paris, Presses de la Renaissance.

- Shyaka Mugabe, Aggée (2009) : Réparation et réconciliation au Rwanda : portée et limites de la justice transitionnelle, Université Catholique de Louvain-la-Neuve. 